\title{
La reconstrucción de la sociología de los intelectuales y su programa de investigación
}

\author{
Juan Pecourt Gracia \\ Universitat de València \\ Juan.Pecourt@uv.es
}

Recibido: 08-10-2014

Aceptado: 13-11-2015

\section{Resumen}

En este artículo, identificaré las principales líneas de investigación que se han desarrollado, a nivel internacional, en el ámbito de la sociología de los intelectuales. Durante mucho tiempo, los debates se restringieron a la reflexión teórica y estuvieron muy relacionados con las luchas ideológicas del campo político. Sin embargo, desde los años ochenta, se ha generado un nuevo campo de investigación que, heredero de los debates del periodo anterior, ha introducido nuevas herramientas teóricas y metodológicas para analizar los contextos y los casos específicos de la acción intelectual. El resultado es un programa de investigación que comparte algunos principios generales, pero que también se caracteriza por incluir diferentes definiciones de su objeto.

Palabras clave: intelectuales; espacio público; campo cultural; campo político

\section{Abstract. Reconstructing the sociology of intellectuals and its research programme}

In this article, the main research paths developed at the international level within the field of the sociology of intellectuals are identified. For a long time, debates remained restricted to theoretical reflection and were clearly linked to the ideological conflicts of the political field. From the eighties onwards, however, a research field taking into account previous debates emerged with the development of new theoretical and methodological tools appropriate for the study of intellectuals in specific social contexts. The result is a research programme which shares some common principles, but at the same time is characterized by different definitions of its object of study.

Keywords: intellectuals; public space; cultural field; political field 


\section{Sumario}

1. Introducción

2. La sociología clásica de los intelectuales

\section{La redefinición del objeto} de investigación
4. La nueva sociología de los intelectuales y sus variantes

Conclusión

Referencias bibliográficas

\section{Introducción}

Presentar un artículo científico sobre la sociología de los intelectuales no es una tarea sencilla. El concepto del intelectual es uno de los más polémicos en el ámbito de las ciencias sociales, mientras que, en la esfera pública, se inserta en un campo semántico que incluye significados muy diversos, algunos de ellos claramente peyorativos. Durante la mayor parte del siglo xx, el debate teórico sobre la función social de los intelectuales fue muy importante (sobre todo en algunos países europeos) y estuvo asociado a las grandes contiendas ideológicas del periodo. Sin embargo, a partir de la década de 1980, el intelectual empezó a considerarse una figura arcaica que no tenía lugar en la nueva sociedad postindustrial y de la que podía prescindirse sin grandes problemas. Los debates teóricos remitieron, del mismo modo que el interés colectivo por sus mensajes.

A simple vista, el desplazamiento del intelectual del debate público implicaría el desmantelamiento de la sociología de los intelectuales como un ámbito específico de investigación dentro de las ciencias sociales. Sin embargo, la tesis fundamental de este artículo es la contraria: a partir de los años ochenta, la sociología de los intelectuales ha tenido un fuerte impulso en el entorno académico, mientras en el ámbito público aumentaba el desinterés o se hablaba directamente de la "desaparición de los intelectuales" (Hollander, 2006; Jacoby, 1987; Lyotard, 1979). La clave de este proceso paradójico se encuentra, por supuesto, en las nuevas formas de entender a los intelectuales que se han desplegado en las últimas décadas. Los debates políticos e ideológicos de antaño han dado lugar a estudios científicos, elaborados con gran esmero metodológico y empírico, que analizan el comportamiento de los intelectuales en contextos sociales diferenciados.

Para desarrollar esta tesis, hemos organizado el artículo en tres grandes apartados. En el primer apartado, abordaremos, de forma muy sintética, los debates teóricos de la sociología clásica de los intelectuales y su relación con las discusiones ideológicas dominadas por el marxismo y el liberalismo. En el segundo apartado, examinaremos la redefinición de los intelectuales como objeto de investigación que se produce a partir de los años ochenta. En este momento, los debates teóricos y fuertemente ideologizados empiezan a sustituirse por estudios empíricos que tratan de comprender la acción intelectual desde una perspectiva científica y, en la medida de lo posible, objetiva. Señalaremos a los sociólogos Pierre Bourdieu y Randall Collins como máximos responsables de este giro teórico y metodológico. Finalmente, en el tercer apartado, identificaremos las principales corrientes de análisis dentro del actual programa de inves- 
tigación de la sociología de los intelectuales, que denominaremos la sociología de los intelectuales consagrados, la sociología de las comunidades intelectuales y la sociología de las intervenciones públicas.

\section{La sociología clásica de los intelectuales}

Después del caso Dreyfus, que origina la presentación pública del intelectual, Benda proporcionó el primer intento de definición del colectivo. En La traición de los intelectuales (1927), puede leerse que los intelectuales son «todos aquellos cuya actividad no persigue esencialmente fines prácticos, sino que, al pretender su felicidad del ejercicio del arte, de la ciencia o de la especulación metafísica, en resumen, de la posesión de un bien no temporal, de alguna manera dicen "Mi reino no es de este mundo"» (Benda, 1927: 123). Los intelectuales, por tanto, comparten unos intereses (de carácter inmaterial o espiritual) que conforman su identidad y los alejan del resto de clases sociales. Benda es el introductor de una temática recurrente en décadas posteriores, la de la decadencia, la muerte o la desaparición de los intelectuales. En su época, coincidiendo con los efectos que el caso Dreyfus tuvo en la sociedad francesa, Benda ya consideraba que los intelectuales eran una especie en peligro de extinción, dominada por las "pasiones políticas» e incapaz de alcanzar las cotas de pureza creativa de las generaciones anteriores, es decir, su pensamiento y sus actuaciones no están guiadas por las leyes de la mente, sino que responden a intereses nacionales, étnicos o de clase. Benda reclamará que los intelectuales ignoren los problemas políticos del momento, que distraen de lo fundamental, para dedicarse al estudio de los temas imperecederos.

Por su parte, K. Mannheim coincide con Benda en que los intelectuales deben desvincularse de los conflictos sociales inmediatos para alcanzar una mayor objetividad, aunque emprende un análisis sociológico bastante más detallado de las comunidades intelectuales. De este modo, introduce su famosa noción del «intelectual desclasado», situado en espacios intersticiales y que no forma parte de ninguno de los grandes bloques sociales en competencia durante la modernidad: la burguesía y el proletariado. Según sus propias palabras: «la actividad intelectual no es privilegio de una clase rigurosamente definida, como el clero, sino más bien un estrato social, en gran parte desligado de cualquier clase social y que se recluta en un área cada vez más extensa de la vida social» (Mannheim, 1934: 193). La intelligentsia está formada por un grupo social autónomo que no responde a los intereses de ninguna de las dos clases y que se articula en torno al interés colectivo de la búsqueda de la verdad. Si Benda considera que los verdaderos intelectuales no forman parte de este mundo, Mannheim realiza un mayor esfuerzo sociológico para explicar la autonomía de los intelectuales teniendo en cuenta el origen diverso de sus integrantes y su compromiso inquebrantable con las leyes del conocimiento. Tanto La traición de los intelectuales de Benda como Ideología y Utopía de Mannheim sitúan la reflexión de los intelectuales en el terreno teórico y no se traducen en investigaciones empíricas específicas. 
Las posiciones de Benda y Mannheim, como decíamos, han sido muy influyentes durante el siglo xx y han conformado las perspectivas liberales del intelectual que desarrollarán autores posteriores, siguiendo parámetros parecidos. Desde el campo marxista, el pensador italiano Antonio Gramsci proporcionará un modelo que entra en plena contradicción con ambas perspectivas. Gramsci considera que los intelectuales no pueden escapar a los intereses materiales de clase, tal como aseguran Benda y Mannheim; forman parte del sistema de clases y su producción cultural está relacionada con los intereses del estrato social en el que se encuentran ubicados. Más concretamente, el marxista italiano afirma que «todo grupo social, como nace en el terreno originario de una función esencial en el mundo de la producción económica, se crea al mismo tiempo y orgánicamente una o más capas de intelectuales que le dan homogeneidad y consciencia de su propia función, no solo en el campo económico, sino también en el social y político» (Gramsci, 1926-1937: 388). Para Gramsci, la idea del «intelectual independiente», subyaciente en las teorías de Benda y Mannheim, es una ficción que no tiene ninguna base en el mundo real. Se trata de una formulación, envuelta en el ropaje del idealismo, que oculta la verdadera labor de los intelectuales al servicio de la ideología social dominante. Frente a la falsedad del intelectual independiente, Gramsci contrapone la noción del «intelectual orgánico», el productor simbólico que es consciente de sus intereses de clase y que prepara el proyecto hegemónico de las clases subalternas con el fin de que estas puedan alcanzar el poder y desplazar a las clases dominantes tradicionales.

$\mathrm{Si}$, antes de la Segunda Guerra Mundial, los debates sobre el papel de los intelectuales en la sociedad moderna se desarrollaron sobre todo en Europa, después de la contienda, los debates norteamericanos adquirirán un peso creciente. Europa capitaneará la defensa del intelectual desde perspectivas marxistas, o muy influidas por el marxismo, mientras que Estados Unidos destacará en las propuestas de carácter liberal, que se realizarán sobre todo desde el funcionalismo (Parsons, 1969; Merton, 1968; Shils, 1972; Coser, 1965; Lipset, 1960). Los funcionalistas retoman la idea de Mannheim según la cual los intelectuales se sitúan en universos relativamente autónomos y, por tanto, no se ven influidos por los conflictos que caracterizan a otros ámbitos de la sociedad (como el político o el económico). Desde su habitual abstracción, Talcott Parsons presenta una formulación paradigmática de esta perspectiva: «son aquellas personas que priorizan el significado de los sistemas simbólicos sobre la interacción con los grupos sociales» (Parsons, 1969: 4). Los funcionalistas critican la orientación revolucionaria de los intelectuales europeos y, siguiendo la tradición de Benda y Mannheim, entienden al intelectual como a un agente autónomo dedicado a la búsqueda desinteresada del conocimiento. La autonomía del intelectual, su alejamiento de la estructura de clases, asegurará su acercamiento a la verdad y a la objetividad. La modernidad impone formas de organización social muy complejas que requieren funciones especializadas, $y$ los intelectuales son precisamente los encargados de realizar las labores simbólicas y normativas requeridas. Dentro de la perspectiva funcionalista, algunos 
incidirán en la profesionalización creciente de las tareas intelectuales (Lipset, 1960; Merton, 1968) y otros insistirán en el carácter sagrado de su misión (Shils, 1972), pero todos ellos estarán de acuerdo en denunciar las posiciones revolucionarias, propias de sociedades ideologizadas que no han alcanzado todavía el equilibrio pragmático de la posguerra. Desde una posición cercana, Daniel Bell también incidirá en la crítica del intelectual marxista, defensor de un proyecto revolucionario para la sociedad, y defenderá la necesidad de intelectuales pragmáticos y desideologizados que se enfrenten a los problemas concretos que plantee la sociedad (Bell, 1960: 393-409).

El contrapeso más importante al consenso ortodoxo del funcionalismo en Estados Unidos lo proporcionaría Charles Wright Mills con su defensa del intelectual comprometido. Mills denunció la alianza de los intelectuales con el establishment político y económico, al tiempo que reclamaba la recuperación de su función crítica y su capacidad de denuncia frente al poder (Wright Mills, 2008). El sociólogo norteamericano identifica la aparición de un aparato cultural (cultural apparatus) que se compone de «todas las organizaciones y los ambientes en los que se realiza el trabajo artístico, intelectual y científico, y en los que se produce y distribuye la información y el entretenimiento" (Wright Mills, 2008: 204). Dentro del entramado institucional, eregido y legitimado bajo el paraguas del funcionalismo, el pensamiento crítico resulta impracticable, porque, en esos entornos burocráticos, los intelectuales han perdido la capacidad de tomar sus propias decisiones. De nuevo, afirma Mills, «los medios de comunicación efectiva están siendo expropiados del trabajador intelectual. La base material de su libertad intelectual y su iniciativa no está en sus manos» (Wright Mills, 2008: 18). La denuncia solitaria de Mills cristalizará años más tarde en los posicionamientos revolucionarios de autores como Chomsky (1969) y Said (1994).

Mientras, en Estados Unidos, el intelectual se desprende de su potencial revolucionario y se integra en las instituciones hegemónicas, en Europa mantendrá la crítica radical al sistema capitalista durante algunas décadas más. Tanto Francia como Italia tienen partidos comunistas muy potentes que son alternativas serias de gobierno, y en torno a ellos prospera una teorización de los intelectuales muy influida por los debates marxistas anteriores a la Segunda Guerra Mundial. Jean Paul Sartre ejemplificaría la figura del intelectual comprometido, que hereda la función crítica de los dreyfusards adaptándola al contexto de la Guerra Fría. El autor de El ser y la nada realiza una síntesis entre el marxismo y el existencialismo y lo presenta como «un producto de sociedades desgarradas, el intelectual da testimonio de ellas, porque ha interiorizado su desgarro. Por lo tanto, es un producto histórico. En este sentido, ninguna sociedad puede quejarse de sus intelectuales sin acusarse a sí misma, pues solo tiene a los que ha hecho" (Sartre, 1972: 41). Si, para Gramsci, los intelectuales son un producto de las clases sociales, para Sartre representan un producto inevitable de la historia y un reflejo de sus contradicciones. El intelectual es un ser esencialmente solitario, desconectado de la burguesía y del proletariado, y abocado a una búsqueda personal, de carácter dialéctico, 
que le remite al mundo (al exterior) y a sí mismo (a su interior). Precisamente esta contradicción, la dualidad entre la introspección y la acción, impulsa su compromiso con los más desfavorecidos de la sociedad. Sartre le atribuye la función precisa de alentar la universalización de las aspiraciones de las clases desfavorecidas (Dosse, 2003: 81). Las ideas de Sartre serán muy influyentes en los intelectuales marxistas europeos de la posguerra.

Sin embargo, el padre del existencialismo también encontrará críticas rotundas a su posición en Francia. La más importante vendrá de la mano de un antiguo compañero de la École Normale Supérieure, el sociólogo Raymond Aron, con la publicación de El opio de los intelectuales (1955). Aron es uno de los teóricos del final de las ideologías y, por tanto, su posición es similar a la de Daniel Bell, su objetivo es criticar las inconsistencias del intelectual revolucionario. Como sugiere el título de su libro, utiliza las herramientas teóricas del marxismo para atacar a los intelectuales marxistas. Si Marx denunciaba que la religión era el opio del pueblo, Aron asegura que el comunismo se ha convertido en una «religión secular» que secuestra la libertad de pensamiento. Aron retorna a Mannheim, aunque, desde una perspectiva sociológica, su análisis es más matizado que el de su predecesor. Según Aron, el «poder espiritual» de los intelectuales puede encontrarse en cuatro subsistemas diferentes: los «hombres de letras», los «académicos», los «expertos» y los «periodistas». Aunque afirma estudiar el fenómeno de los intelectuales desde una cierta neutralidad y acepta el papel de los cuatro colectivos dentro del poder espiritual de la sociedad moderna, finalmente adopta una posición normativa que considera a los creadores inmersos en la producción del «conocimiento por el conocimiento" como los verdaderos intelectuales. La separación entre los verdaderos intelectuales y los demás productores culturales se sitúa en «una zona vaporosa donde los popularizadores dejan de interpretar y comienzan a desorientar, y donde, guiados solamente por el éxito y por el dinero, esclavos de los gustos supuestos de su público, se desprenden de los valores que dicen defender» (Aron, 1955: 255).

Mientras en Occidente se produce el choque entre los intelectuales liberales y los intelectuales marxistas, en los países situados bajo la órbita soviética surge el debate sobre la «nueva clase», que tendrá hondas repercusiones y renovará las definiciones del intelectual. Aunque el concepto es originario de Bakunin (1870), la popularización de esta idea se debe al disidente yugoslavo Milovan Djilas (1957), que denunciaba que la Unión Soviética y los países de Europa del Este habían renunciado al ideal de la igualdad mediante el establecimiento de una nueva clase formada por burócratas privilegiados del Partido Comunista (en la que no incluía a los intelectuales). Posteriormente, se reformuló la idea de la nueva clase y se situó a los productores de conocimiento, y no a los burócratas, en el centro de la administración socialista (Gella, 1976; Konrad y Szelenyi, 1979).

La teoría de la nueva clase sufrió una segunda reformulación cuando estas ideas empezaron a utilizarse en Occidente en las décadas de 1960 y 1970 con diferentes efectos ideológicos. Desde el liberalismo, Daniel Bell (1973) adoptó 
algunas de estas ideas en su teoría de la sociedad postindustrial, caracterizada por la importancia del conocimiento teórico en detrimento de la producción material. El intelectual de Nueva York escribe: «en la sociedad postindustrial, el problema clave es la organización de la ciencia y la institución primordial la universidad o los institutos de investigación donde se lleva a cabo semejante labor» (Bell, 1973: 143). La nueva clase estaría compuesta sobre todo por intelectuales científicos, considerados agentes clave del desarrollo económico y social. Desde el marxismo, Alvin Gouldner (1979) proporciona una interpretación muy diferente, al otorgar a la nueva clase un papel revolucionario, en sustitución de la vieja clase del proletariado. Según el sociólogo norteamericano, la nueva clase estaba compuesta por dos colectivos diferentes, los «intelectuales críticos» y la «intelligentsia técnica», que compartían una misma "cultura del discurso crítico», es decir, un conjunto de reglas que $a$ ) se preocupa por justificar sus proposiciones básicas, pero $b$ ) su modo de justificación no se basa en la apelación a las autoridades, sino que prefiere obtener el consenso voluntario de aquellos a los que se dirige en base a la argumentación (Gouldner, 1979: 28). Desde su punto de vista, la "cultura del discurso crítico» se estaba constituyendo como el elemento diferenciador de la nueva clase frente a las viejas clases sociales. Se distancia, por tanto, de la postura liberal de Bell, que encuentra a los intelectuales en el núcleo de la producción burguesa, y también de la posición marxista de Gramsci, que sitúa a los intelectuales orgánicos en el seno de la clase trabajadora.

\section{La redefinición del objeto de investigación}

A partir de los años ochenta, las teorías marxistas y funcionalistas, así como las teorías vinculadas a la nueva clase, perdieron relevancia en un momento de crisis y recomposición en el ámbito de las ciencias sociales (Gouldner, 1970). Frente a la ambición y a las pretensiones universales de las teorías precedentes, los nuevos planteamientos suelen ser, en general, más modestos y localizados, tienden a cuestionar la transcendencia tradicionalmente otorgada a los intelectuales. Los ejemplos más conocidos del cambio de orientación son las propuestas del «intelectual específico» de Foucault (1980), entendido como un actor especializado que interviene exclusivamente en los asuntos acerca de los que tiene un conocimiento técnico contrastado, y el «intelectual intérprete» de Bauman (1987), un agente propio de las sociedades multiculturales que, en lugar de emitir juicios inapelables o verdades universales, asume la misión de facilitar la comunicación entre comunidades con pautas culturales diferenciadas. Junto a la reducción del alcance de la acción intelectual, en este periodo, también aparecen diversos trabajos que cuestionan su relevancia social (Jacoby, 1987; Lyotard, 1984) e inciden en sus tendencias totalitarias (Ferry y Renaut, 1985; Judt, 2007) o en la sordidez de sus vidas privadas (Johnson, 1989).

Sin embargo, mientras, en el terreno mediático y social, se cuestiona la misión profética del intelectual, en el ámbito sociológico han surgido corrientes de pensamiento que quieren dotar de un mayor rigor científico a las antiguas 
ideas y, de este modo, generar nuevos espacios de investigación. De las teorías clásicas, se heredan las problemáticas generales, pero tienden a abandonarse los instrumentos específicos para abordarlas, tanto los marcos conceptuales como las estrategias metodológicas, que se consideran insuficientes para una comprensión adecuada del fenómeno estudiado. Diferentes observadores (Kurzman y Owens, 2002; Gross, 2008; Picó y Pecourt, 2008; Vázquez García, 2009; Eyal y Buchholz, 2010; Baert e Isaac, 2011) coinciden en señalar a los sociólogos Pierre Bourdieu y Randall Collins como las referencias más importantes de las nuevas tendencias de investigación. Desde nuestro punto de vista, efectivamente, los trabajos de Bourdieu y Collins son fundamentales en la reconstituida sociología de los intelectuales, porque impulsan tres desplazamientos metodológicos básicos. En primer lugar, proponen una transición desde los tratados eminentemente teóricos, que no tienen ninguna repercusión empírica directa, hasta los estudios que combinan las aportaciones teóricas con el análisis detallado de las comunidades y las trayectorias individuales. En segundo lugar, replantean la posición social del intelectual, desde las polémicas recurrentes sobre su inserción en el sistema de clases (o también en el seno de proyectos nacionales) hasta su ubicación en universos específicos que funcionan de acuerdo con normativas autónomas. En tercer lugar, y en relación con la cuestión anterior, ambos autores no se interesan tanto por la función social de los intelectuales (un tema obsesivo de las generaciones anteriores) como por las estrategias específicas que emplean para mejorar su situación dentro de las propias comunidades de origen.

En las siguientes páginas, analizaremos cómo estos desplazamientos básicos en la sociología de los intelectuales se han concretado en propuestas teóricas concretas y, también, en estudios empíricos de redes intelectuales. Primero, sintetizaremos las aportaciones básicas de Bourdieu y Collins a la sociología de los intelectuales, incidiendo en los conceptos centrales que guían sus investigaciones, resultado de las rupturas metodológicas que acabamos de subrayar. Recurriendo al lenguaje metodológico de Lakatos, podríamos considerar que ambos forman parte del «núcleo firme» del programa de investigación de la sociología de los intelectuales. Más adelante, veremos cómo estas ideas se han concretado en diversas rutas de investigación que tienen una cierta homogeneidad (por ejemplo: existe un acuerdo común acerca de la necesidad de combinar el trabajo teórico con la investigación empírica), pero que también presentan en su seno una diversidad de corrientes y de objetos de investigación.

\section{La aportación de Bourdieu}

Bourdieu sitúa a los intelectuales en el núcleo de su producción sociológica. De todas formas, Bourdieu no reproduce las ideas de la sociología clásica de los intelectuales, sino que trata de analizar este colectivo social de acuerdo con unas coordenadas teóricas que pretenden corregir las deficiencias de sus predecesores. Sus ideas reaccionan contra las limitaciones del funcionalismo (Parsons, Merton, Shils), que suele entender las comunidades intelectuales como 
entes idealizados, y también contra las restricciones del marxismo (Gramsci, Sartre), que establece una conexión directa entre las comunidades intelectuales y el sistema de clases. En su lugar, partiendo de la diversificación de las tareas intelectuales delineada por Raymond Aron (1955), sitúa a los productores simbólicos en diferentes campos culturales y considera que su definición e identidad dependen de su posición en estos espacios. El análisis estructural de los campos culturales propuesto por Bourdieu abrirá un horizonte inédito en el estudio sociológico de la inteligencia que ha tenido múltiples seguidores, como veremos más abajo. Podemos sintetizar las ideas fundamentales del sociólogo francés en los siguientes puntos:

a) Los campos culturales, del mismo modo que los campos políticos y económicos, son arenas de conflicto en donde se dilucidan las propuestas culturales legítimas, es decir, se resuelve qué elementos (autores, obras, ideas) serán incluidos o excluidos del canon cultural (artístico, literario, científico, etc.). De acuerdo con esta lógica de inclusión y exclusión, identifica una oposición básica entre dos sectores, los «establecidos» (ortodoxos) y los «aspirantes» (heterodoxos), que condicionará las prácticas intelectuales (Swartz, 1997: 226). Establece, así, un vínculo directo entre la posición dentro del campo cultural (caracterizada por una autonomía variable) y el contenido específico de las obras (definidas por su mayor o menor innovación).

b) Dentro de la teoría de los campos culturales, Bourdieu identifica dos grandes mercados destinados a la producción y al consumo cultural que darán lugar a tipologías diferenciadas de intelectuales: por una parte, el campo cultural de masas y, por la otra, el campo cultural especializado. Los mercados especializados tienden a estructurarse alrededor de formas autónomas de poder simbólico frente a poderes políticos y económicos. Son los mercados de la «ciencia pura» o del «arte por el arte», generadores de productos simbólicos de difícil comprensión para el público no iniciado (Bourdieu, 1995). En cambio, los espacios de producción cultural masificados están orientados hacia criterios externos, como el éxito comercial o la demanda popular, es decir, producen objetos simbólicos que pueden transformarse rápidamente en capital económico.

c) Bourdieu asegura que los comportamientos individuales se rigen por la «ley de la búsqueda de la distinción» (Bourdieu, 1971: 35). Aunque se trate de un principio universal, la lucha por la distinción es especialmente intensa entre los intelectuales, dado que, para este colectivo, «existir es diferir, es decir, ocupar una posición distintiva en el campo cultural» (Bourdieu, 1983). Al mismo tiempo, según Bourdieu, para tener éxito en el campo cultural y, de este modo, lograr una posición distinguida, el individuo necesita dotarse de recursos específicos, sobre todo de formas diversas de capital cultural (habilidades y conocimientos) y simbólico (reconocimiento), así como de un habitus apropiado, o de un conjunto de disposiciones interiorizadas, que le permita interpretar intuitivamente las reglas del campo.

d) Aunque Bourdieu incide en el estudio de las dinámicas internas de los campos culturales y en las estrategias prácticas de los participantes, no se olvida 
de la conexión de estos espacios con el mundo exterior. La relación entre los diferentes campos que conforman la sociedad también se caracteriza por la desigualdad y las relaciones de poder. Los campos dominantes se encuentran en las esferas políticas y económicas, constituidas como fortalezas que monopolizan los recursos más valiosos de la sociedad y que tienden a imponer sus normas y reglas sobre el resto, mientras que los dominados se encuentran, entre otros sectores, en la esfera cultural. El análisis de Bourdieu permite replantear la cuestión de la autonomía del intelectual desde las luchas históricas encaminadas a la construcción de campos culturales independientes de los grandes poderes sociales. Por esta razón, el sociólogo francés apela a un "corporativismo de lo universal» que permita a los intelectuales asociarse y actuar desde campos de acción autónomos.

\section{La aportación de Collins}

En cierta medida, las ideas de Randall Collins pueden interpretarse como un intento de establecer un contrapeso al influjo teórico de Bourdieu. Siguiendo la estela de R. Dahrendorf y L. Coser, el profesor de la Universidad de Pennsylvania acude a la sociología del conflicto para contrarrestar la visión armónica de las comunidades intelectuales que tienden a imponer los máximos representantes del funcionalismo (Collins, 1975). Por otro lado, también se muestra crítico con la interpretación marxista del conflicto, centrada en la lucha por el capital económico, por no tomar en consideración la importancia de los recursos inmateriales que condicionan las desigualdades sociales (Joas y Knöbl, 2009: 187). Si lo comparamos con Bourdieu, que centra su estudio en las dinámicas internas y externas de los campos culturales, Collins prefiere estudiar las interacciones que se producen entre intelectuales en el ámbito microsociológico (en clases, reuniones, seminarios, congresos, etc.). Estos ámbitos de interacción, que suelen quedar fuera de los grandes tratados históricos y sociológicos, centrados en el comentario de las grandes teorías, son los entramados sociales que determinan el éxito o el fracaso de las ideas. Collins se interesa por los rituales específicos de los intelectuales, y por los recursos inmateriales asociados a ellos, para asignar valor social a sus obras. De forma sintética, resumiremos la posición de Collins en los siguientes puntos:

a) Collins sustenta el estudio de los intelectuales en la idea del «ritual de interacción». Asegura que el análisis de los campos de Bourdieu, a pesar de los esfuerzos de su autor, se sitúa en un plano macroestructural y no tiene en cuenta las interacciones cotidianas que conforman los diferentes submundos de la cultura. Por ello, prefiere centrarse en los rituales específicos de la inteligencia, producidos en contextos de interacción cara a cara, que construyen la identidad de sus miembros y permiten elaborar los «símbolos abstractos descontextualizados" (Collins, 1998: 25) que fundamentan la actividad intelectual. Estas microinteracciones cara a cara darán lugar a las macroestructuras sociales mediante su encadenamiento sucesivo, es decir, los rituales intelectuales 
se suceden de forma progresiva, estableciéndose regularidades y tendencias a nivel macrosociológico.

b) Del mismo modo que Bourdieu, Collins asegura que, para participar en las redes intelectuales, se necesitan recursos específicos. Comparte con el autor francés la importancia del «capital cultural» (que, en algunas ocasiones, denomina "símbolos de membresía»), pero, al mismo tiempo, dota de una importancia fundamental a las emociones, a las que se refiere con el término de «energía emocional». Para Collins, los rituales de interacción cara a cara suscitan un estado colectivo de «efervescencia emocional» que transmite a los participantes emociones positivas como la confianza, el entusiasmo o el placer, ingredientes básicos que permiten elaborar proyectos originales, creativos y rupturistas. Dentro de las comunidades intelectuales, no todo el mundo puede acumular la energía emocional necesaria para realizar un trabajo ambicioso y sostenido en el tiempo; más bien al contrario, las emociones positivas se distribuyen de forma muy desigual: una minoría creativa que tiende a monopolizarla y una mayoría acomodaticia y poco productiva que no tiene acceso a ella.

c) Collins asegura que la condensación de energía emocional producida en los rituales exitosos puede provocar la aparición de «objetos sagrados» venerados por la comunidad. Considera que los escritores y los filósofos son actores situados en mercados de interacción particulares, dedicados a la confección de un tipo determinado de "objeto sagrado». Este objeto es un producto simbólico (una teoría, una idea, un principio, una fórmula) creado en una comunidad que se define por rituales y criterios de sacralidad basados en una cierta noción de "excelencia». A través de sus interacciones ritualizadas, los intelectuales crean símbolos abstractos que distribuyen bajo la denominación de «verdad», «objetividad» o "ciencia», dotados de una sacralidad especial que a veces no se percibe desde el exterior (Collins, 1998: 24).

d) Un elemento determinante de la estructura de las redes intelectuales es la estratificación del «espacio de atención» y la consiguiente desigualdad en las posibilidades de ser escuchado (Collins, 1975, 1998). La comunidad tiene una forma de pirámide: no todos los participantes están situados en el mismo nivel ni todas las interacciones tienen la misma relevancia. Muchos participantes del mundo intelectual son participantes ocasionales que no tienen un peso relevante y pasan prácticamente desapercibidos. Dentro de esta divisoria, el grupo selecto que domina las formas de prestigio y reconocimiento supone entre el 1 y el 2 por ciento del total de la comunidad. Esta jerarquización del espacio de atención da lugar a lo que R. Collins denomina «ley de los pequeños números» (Collins, 1998: 42-44). El autor norteamericano llega a cuantificar entre 3 y 6 el umbral de las posiciones realmente relevantes dentro de la comunidad intelectual.

\section{La nueva sociología de los intelectuales y sus variantes}

Tanto en Europa como en Estados Unidos, acabado el periodo de las grandes teorías sobre el intelectual, la emergencia de nuevas perspectivas, dotadas de 
mayor precisión metodológica y contenido empírico, ha impulsado un programa de investigación muy fructífero. El influjo de Bourdieu es omnipresente en ambos continentes y todos los autores localizados en este espacio de investigación necesitan gestionar dicha influencia. Los conceptos de "campo cultural», «capital cultural», "capital simbólico» y «habitus» han demostrado tener una gran fuerza de atracción. La influencia de Collins no está tan generalizada (así, por ejemplo, no tiene una presencia preponderante en el ámbito francés), pero su importancia es creciente. El diferencial entre ambos se encuentra en la potencia explicativa que, hasta el momento, han alcanzado sus respectivas propuestas. Posiblemente, una de las debilidades de Collins se esconde en el concepto de «energía emocional», criticado por ambigüedad y superficialidad teórica (Misztal y Freundlieb, 2003: 251). Aún así, en su trabajo, Collins insiste en la productividad empírica del concepto. Más allá de sus diferencias, ambos sociólogos representan los principios básicos que estructuran este subcampo científico, entre ellos, la necesidad de combinar la reflexión teórica con el trabajo empírico o el replanteamiento de las problemáticas clásicas sobre la posición social y la función social del intelectual.

De un modo más específico, Frickel y Gross (2005), en su trabajo sintético sobre los movimientos científico-intelectuales, y bajo la influencia evidente de Bourdieu y Collins, presentan cuatro proposiciones básicas que, desde nuestro punto de vista, explicitan los fundamentos básicos de la nueva sociología de los intelectuales. La proposición 1 entronca con la sociología clásica de los intelectuales y reivindica la relevancia de los intelectuales de estatus elevado, a los que denominan «innovadores conceptuales». La proposición 2, claramente influida por las problemáticas que plantea Bourdieu, señala la importancia de las "condiciones estructurales para acceder a los recursos esenciales». La proposición 3 recoge las aportaciones de Collins e insiste en el papel que adquieren los «contextos de micromovilización». Finalmente, la proposición 4 pone el acento en la necesidad de que las ideas adquieran resonancia en la comunidad intelectual (Frickel y Gross, 2005: 209-225). Estas proposiciones teóricas establecen las coordenadas básicas que han orientado a las investigaciones empíricas sobre los contextos localizados de la acción intelectual.

Por supuesto, el nuevo programa de investigación no es hermético ni completamente homogéneo, puesto que pueden detectarse definiciones divergentes del objeto de investigación. Acompañando los principios del «núcleo firme», también se observan las influencias complementarias de Anthony Giddens, Gary Becker, Jeffrey Alexander, Norbert Elias, Michel Foucault, Harold Garfinkel o Bruno Latour, así como referencias interdisciplinarias procedentes de la historia social de las ideas (Hayden White, Dominick LaCapra y David Harlan), la historia de los conceptos (John Pocock, Quentin Skinner, John Dunn y Reinhart Koselleck) o la filosofía (Richard Rorty y John Austin). En beneficio de la claridad y de la parsimonia de la argumentación, sintetizaremos estas corrientes en tres grandes frentes de investigación. En primer lugar, los trabajos que toman como objeto a individuos carismáticos dentro del campo intelectual (sociología de los intelectuales consagrados); en segundo lugar, las pro- 
puestas enfocadas hacia el conjunto de la comunidad intelectual (sociología de las comunidades intelectuales), y, finalmente, en tercer lugar, los estudios de los nuevos espacios y de las nuevas modalidades de acción intelectual (sociología de las intervenciones públicas).

\section{La sociología de los intelectuales consagrados}

Algunos autores utilizan las innovaciones de la sociología de los intelectuales para estudiar las trayectorias de intelectuales influyentes dentro de sus contextos específicos de acción. Las aproximaciones que siguen esta orientación abordan autores muy diversos, como serían los casos de Marx (Gouldner, 1985), Heidegger (Bourdieu, 1988), Sartre (Boschetti, 1995), Mozart (Elias, 2002) y Jesús Ibáñez (Moreno Pestaña, 2008), y se apoyan en diferentes metodologías y marcos conceptuales. Entre todas estas aportaciones, destacan las que proceden de la denominada «nueva sociología de las ideas", una corriente de investigación renovadora dentro de la sociología de los intelectuales que incluye autores como Michelle Lamont, Charles Camic y Neil Gross. Además de las referencias centrales que suponen los trabajos de Bourdieu y Collins (Camic, 2001), sus representantes están muy influidos por las innovaciones producidas en el ámbito de la historia intelectual anglosajona, y especialmente por los trabajos metodológicos de la Escuela de Cambridge (John Dunn, Quentin Skinner, John Pocock). Los renovadores contextualistas toman de los historiadores de Cambridge el supuesto de que las ideas no son «transparentes». Para estudiarlas, hay que situarlas en los contextos sociolingüísticos en las que fueron producidas, y comprender así la intención específica del autor (Skinner, 2002). El significado original de las obras solo puede obtenerse relacionándolas con el conjunto de textos que componen su espacio lingüístico y contra los que, de alguna manera, reacciona y se posiciona. Estos espacios pueden reconstruirse con un examen riguroso de los textos producidos por los diferentes autores en un determinado momento histórico, mediante un análisis lingüístico que extraiga el sentido dado a los conceptos políticos fundamentales. Los autores de la Escuela de Cambridge utilizan esta metodología para estudiar a los clásicos de la teoría política (Hobbes, Maquiavelo, Locke, etc.) y, de este modo, evalúan sus innovaciones respecto a otros textos de su época. Por ejemplo, Skinner (2008), en su estudio de la obra de Hobbes, reconstruye el contexto local en el que se situaba Hobbes, con sus autores y sus obras, y trata de entender qué pretendía hacer exactamente al escribir Leviatán. Skinner entiende a Hobbes como un «ideólogo innovador» que utiliza su virtuosismo retórico para dotar de un nuevo significado a los conceptos políticos establecidos (por ejemplo, asegura Skinner, Hobbes redefine la idea de libertad en el contexto del absolutismo monárquico).

Los estudios de Lamont, Camic, Gross y otros recogen las enseñanzas metodológicas de la Escuela de Cambridge y las adaptan al programa de investigación de la nueva sociología de los intelectuales inspirada por Bourdieu y Collins. En su caso, tratan de realizar estudios empíricos localizados de las trayectorias de 
individuos especialmente influyentes dentro del campo cultural. En el mundo moderno, los contextos locales en los que se dirimen las batallas conceptuales suelen ser las disciplinas académicas, por lo que su foco de atención suele situarse en estos espacios; del mismo modo que Skinner y su análisis de los «ideólogos innovadores» se centran en trayectorias de individuos distinguidos, analizadas desde una perspectiva sociológica que pretende reconstruir el espacio de posibilidades teóricas en el que están instalados. El trabajo de Michelle Lamont, catedrática de la Universidad de Harvard, sobre la trayectoria de Jacques Derrida en el campo académico norteamericano es un claro precedente de esta tendencia. Lamont (1987) trata de comprender las estrategias utilizadas por Derrida para alcanzar una posición predominante en los departamentos norteamericanos de literatura. Identifica tres elementos fundamentales que determinan su éxito: en primer lugar, Derrida logra un estilo retórico propio e inconfundible que lo distingue del resto de competidores. Su estilo se caracteriza por combinar elementos procedentes de las metodologías científicas de las ciencias sociales con otros que se originan en el ámbito de la literatura vanguardista. En segundo lugar, el filósofo francés se sitúa en una tradición filosófica de gran prestigio (procedente de Heiddeger, Husserl y Nietzsche), algo que permite catapultarlo a la cima intelectual. En tercer lugar, Derrida tiene la habilidad de empaquetar su pensamiento mediante conceptos marca (por ejemplo: la idea de la «deconstrucción») que facilitan enormemente su difusión en la comunidad académica y, también, en la población letrada más amplia.

Además de Lamont, Camic y Gross también han realizado análisis contextualizados sobre las trayectorias académicas de intelectuales influyentes. Por una parte, Camic se ha centrado en el trabajo del sociólogo funcionalista Talcott Parsons, el referente central de la sociología teórica hacia la mitad del siglo xx. Según él, durante la concepción de La estructura de la acción social (1968), Parsons tenía como objetivo establecer la sociología como una disciplina científica que fuera plenamente reconocida dentro del mundo académico. Para lograrlo, tuvo que realizar una serie de movimientos estratégicos que lo llevaron a acercarse a determinadas referencias teóricas y alejarse de otras. Así, por ejemplo, Camic $(1987,1992)$ asegura que la orientación teórica de Parsons era perfectamente compatible con el institucionalismo (Veblen, Hamilton y Ayres); sin embargo, tuvo la gran precaución de alejarse de esta corriente, porque estaba mal vista entre sus colegas del Departamento de Economía de Harvard, y se acercó a aquellos autores que estaban bien vistos en aquel momento (Marshall, Pareto, Weber y Durkheim). Dejarse influir por los outsiders hubiera supuesto mayores dificultades para la institucionalización de la sociología en la universidad norteamericana. Por otra parte, Gross (2008) también propone un análisis contextualista en su estudio de la trayectoria académica del filósofo pragmático Richard Rorty, en la que trata de comprender su ruptura con la filosofía analítica, la corriente dominante de su periodo formativo. El análisis contextual de Gross introduce la idea del «autoconcepto del intelectual», entendido como «la totalidad de las ideas y de los sentimientos de un pensador que hacen referencia a sí mismo como intelectual» (Gross, 2008: 312). La idea 
que el intelectual tiene sobre sí mismo (de quién es) determinará sus acciones y el contenido de sus obras. De esta forma, Gross asegura que la socialización familiar de Rorty, en la que sus padres le inculcaron una visión liberal y pragmática del mundo, determinó que, años más tarde, rompiera abruptamente con la filosofía analítica, ya que esta corriente de pensamiento era incompatible con la autoconcepción que Rorty se había creado a lo largo de los años y que culminaría con la publicación de su conocido tratado La filosofía y el espejo de la naturaleza (1989).

\section{La sociología de las comunidades intelectuales}

Además del estudio de casos individuales, podemos identificar una segunda tendencia que centra su atención en el conjunto de la comunidad intelectual. En este caso, el interés se encuentra en el análisis de la organización comunitaria, en sus dinámicas y en sus procesos internos de transformación. Pueden encontrarse precedentes tanto en el análisis sociológico de los «círculos intelectuales» de Kadushin (1974) como en los estudios historiográficos, pero influidos por las ciencias sociales, de Ringer (1969) y Lepenies (1994). Los representantes del giro teórico que hemos comentado en el apartado anterior, Bourdieu y Collins, han orientado sus trabajos más importantes en esta dirección. En ellos, ponen en funcionamiento sus planteamientos teóricos y metodológicos y ofrecen, como resultado, análisis empíricos muy potentes que abren nuevos horizontes a la sociología de los intelectuales. Si Bourdieu se centra en la posesión de recursos específicos y en su relación con la posición en el campo cultural, Collins prefiere indagar en los contextos de micromovilización que posibilitan las comunidades intelectuales.

Por una parte, Bourdieu $(1984,1995)$ centra sus estudios empíricos, en los que combina diferentes técnicas de investigación (cuantitativas y cualitativas) pero donde suele predominar el análisis estadístico, en diversas comunidades intelectuales parisinas (desde los colectivos artísticos del siglo xix hasta las disciplinas académicas previas a la revolución del 68). En todos los casos, muestra cómo sus representantes luchan por el reconocimiento simbólico, y de qué forma su éxito depende de poseer capital cultural e incorporar un habitus adecuado. Los campos culturales suelen ser relativamente estables hasta que, en un momento de crisis, los intelectuales heréticos, en principio alejados de las pugnas por el reconocimiento, logran cambiar las reglas del juego (como lo harán los impresionistas - Manet, Pisarro y Degas - en el mundo del arte, y los estructuralistas - Levy-Strauss, Althusser y Barthes - en el ámbito universitario). Paralelamente a las luchas internas por el reconocimiento, Bourdieu identifica los conflictos externos que aspiran a lograr la autonomía del campo cultural frente a los grandes poderes sociales. A este respecto, resulta de gran interés su análisis de la conquista de la autonomía del campo artístico y literario parisino a lo largo del siglo XIX.

Por otra parte, Collins $(1975,1998)$ también considera que las comunidades intelectuales están altamente estratificadas. El sociólogo norteamericano, 
mediante una investigación de tipo documental alejada del análisis estadístico ofrecido por Bourdieu, realiza un análisis global de las grandes comunidades intelectuales a lo largo de la historia (en Europa, Norteamérica, India, China y Japón). Mediante la comparación de casos diversos, identifica una serie de pautas generales (que, utilizando un lenguaje de corte cuantitativista, denomina leyes) que caracteriza a todas ellas, más allá de las tradiciones específicas que las compongan. Por una parte, Collins incide en la importancia de las redes intelectuales ( $y$, en especial, de las relaciones entre maestro y discípulo) a la hora de asegurarse la preeminencia en el mundo intelectual y la influencia en las generaciones venideras. Por ejemplo, Wittgenstein aprovechó sus conexiones con las grandes escuelas filosóficas de la época (la filosofía analítica de Russell y Whitehead, y el positivismo lógico de Carnap y Frege) para situarse en el centro de atención del campo filosófico. Por otro lado, a diferencia de Bourdieu, que se centra en el reconocimiento simbólico, Collins insiste en los factores emocionales que determinan el éxito o el fracaso intelectual. En el caso de Wittgenstein, de nuevo, lo presenta como un "vampiro emocional» que tiene la capacidad de absorber las energías creativas de la gente que le rodea en beneficio de su propio proyecto intelectual.

Otros autores han presentado detallados análisis empíricos (generalmente basados en la investigación documental) de diversas comunidades intelectuales. En este ámbito, las herramientas conceptuales de Bourdieu han sido especialmente influyentes en investigaciones sobre el campo intelectual francés (Charle, 1990; Kauppi, 1996; Sapiro, 2009a), el campo académico norteamericano (Lamont, 2010), el campo académico argentino (Rubinich, 2001), así como el campo filosófico y cultural español (Pecourt, 2008; Vázquez García, 2009; Moreno Pestaña, 2013). Un trabajo destacado en esta parcela, debido a su meticulosidad empírica, es el estudio publicado por Rémy Rieffel (1993) sobre las redes intelectuales parisinas desde la Segunda Guerra Mundial. Su propuesta se articula en tres planos diferentes: en primer lugar, presenta los «modos de afiliación» de los intelectuales a través de espacios públicos y privados, coloquios, encuentros, militancias, etc. En segundo lugar, define los «modos de legitimación» de los intelectuales a través de las revistas, que otorgan los verdaderos certificados de pertenencia a la comunidad. Y, en tercer lugar, muestra los «modos de consagración» intelectual, mediante su aceptación en grandes instituciones, la publicación en editoriales de prestigio, etc.

Bourdieu y sus seguidores han sido criticados por situar los campos sociales en el contexto del Estado nación, sin tomar en consideración la creciente interdependencia de la sociedad global. Por el contrario, Collins tiene como objetivo prioritario realizar una sociología global de los intelectuales, por lo que podría tomarse como referencia para futuros trabajos sobre la internacionalización de las comunidades intelectuales. Algunos estudiosos como Charle (1996), Casanova (2003) y Sapiro (2009b) están empezando a transitar por este camino. Uno de los trabajos más influyentes sobre la interconexión global de las comunidades intelectuales es French theory (2008), del francés François Cusset, que investiga cómo la universidad norteamericana, en un momento 
de crisis en las humanidades, incorporó las teorías estructuralistas desarrolladas en Francia para recomponer el espacio de investigación. Cusset muestra cómo se agruparon conceptos diversos, de autores variopintos (Deleuze, Foucault, Derrida y Baudrillard), para generar nuevas etiquetas académicas que han tenido una gran influencia posterior (genealogía, deconstrucción, postmodernismo, etc.). El sentido original de las teorías generadas por los autores estructuralistas se transforma en beneficio del marco universitario americano y de su adaptación a proyectos políticos contrapuestos (desde las políticas de la identidad hasta el neoconservadurismo).

\section{La sociología de las intervenciones públicas}

Los espacios de investigación que acabamos de comentar, uno orientado hacia el estudio de casos individuales y otro hacia las estructuras colectivas, comparten diversos supuestos teóricos procedentes de los debates clásicos sobre el intelectual. De este modo, las nuevas aproximaciones establecen tipologías estrictas que diferencian al «intelectual» del «no intelectual», al tiempo que sitúan a los intelectuales en espacios altamente especializados. Por un lado, la sociología de los intelectuales consagrados se centra en individuos carismáticos dentro de sus áreas de conocimiento y, por el otro, la sociología de las comunidades intelectuales suele abordar las reglas de funcionamiento que tienen los universos culturales tradicionales (científicos, filosóficos, literarios, artísticos, etc.). Esta concepción tan restrictiva suele asociarse con las tesis sobre la decadencia del intelectual, citándose como responsables los nuevos espacios sociales que compiten con los tradicionales, como los medios de comunicación (Debray, 1979; Boudon, 1981; Bourdieu, 1997) o los campus académicos (Jacoby, 1987; Collins, 1998; Posner, 2001).

Sin embargo, frente a las definiciones más ortodoxas, se han realizado propuestas que analizan las nuevas configuraciones intelectuales caracterizadas, entre otros factores, por ocupar nuevos espacios y desarrollar nuevas modalidades de actuación. Eyal y Buchholz (2010) aseguran que la sociología clásica de los intelectuales parte de definiciones muy estrictas del intelectual y no tiene en cuenta los espacios intersticiales y las múltiples modalidades de participación. Para superar estas restricciones, proponen el desarrollo de una «sociología de las intervenciones públicas», donde «la intervención no es entendida como una osada zambullida desde un mundo (tranquilo, académico) hasta otro mundo diferente (agonístico, político), sino que tiene lugar en el campo intersticial de los expertos, donde la diferencia entre ambos mundos se difumina» (Eyal y Buchholz, 2010: 132). Lo importante, por tanto, no es definir a un determinado actor social perteneciente a una comunidad cultural independiente y que trata de intervenir en los asuntos públicos, sino resaltar los contornos de un agente heterogéneo que utiliza un determinado conocimiento experto (de orígenes muy diversos) para intervenir en la esfera pública (de formas muy diversas).

Por una parte, encontramos investigaciones que detallan empíricamente las diferentes formas de intervención pública, más allá del compromiso político 
clásico (Epstein, 1996; Keck y Sikkink, 1998). En este campo, destaca el trabajo de la socióloga francesa Giselle Sapiro (2009a) y la relación que establece entre las formas de compromiso público y la posición en el campo intelectual. Las posibilidades de intervención pública, según ella, se articulan en torno a tres ejes: a) la posesión de capital simbólico, que conllevará modelos de intervención individuales o colectivos; $b$ ) la independencia respecto a fuerzas externas, que condicionará la autonomía del formato discursivo, y $c$ ) el nivel de especialización, que supondrá realizar intervenciones en el contexto de cuerpos profesionales organizados o de manera más autónoma. De acuerdo con estos ejes estructurantes, Sapiro identifica siete modelos de intervención pública que conforman diferentes tipos de intelectuales: el «intelectual universal», el "guardián del orden moral», el «intelectual vanguardista», el «intelectual político», el «intelectual experto», el «intelectual específico» y el «intelectual colectivo». En Estados Unidos, Richard Posner propone también una tipología de intervenciones intelectuales dependientes de las leyes de la oferta y la demanda que operan en el «mercado de los productos intelectuales». El análisis empírico de Posner identifica diversos géneros y formatos textuales que moldean las intervenciones en la esfera pública: el «comentario a tiempo real», el "comentario profético», la «jeremiada», la "crítica social general», la "crítica social específica», el «reformismo social», la "crítica político-literaria», la «sátira política» o el «testimonio de expertos» (Posner, 2001)

Por otra parte, nos topamos con investigaciones centradas en la estructura y en la dinámica de los nuevos espacios de la acción intelectual, como los medios de comunicación de masas o los think tanks. Así, en un original trabajo, Jacobs y Townsley (2011) estudian la formación del «espacio de la opinión» en Estados Unidos, entendido como «una parte especialmente influyente de esfera pública política y de élite, esto es, una parte de la infraestructura comunicativa pública donde las elites de las sociedades complejas debaten sobre temas de interés general» (Jacobs y Townsley, 2011: 13). Los autores cuestionan las denuncias del «intelectual mediático» lanzadas por Bourdieu y otros, porque, según ellos, no sustentan su crítica en investigaciones empíricas detalladas. Por tanto, en su trabajo, tratan de estudiar el complejo entramado de relaciones sociales, actores, instituciones y tradiciones culturales que componen el espacio de la opinión en Estados Unidos. Para determinar su estructura, se centran en dos cuestiones básicas: la cuestión del «acceso», basada en el equilibrio que se establece entre insiders y outsiders del campo periodístico, y la cuestión de la "autonomía», dependiente de la capacidad del individuo para hablar por sí mismo, y no en representación de otros intereses sociales. Frente a las tesis de la decadencia del intelectual, Jacobs y Townsley concluyen que el espacio de opinión se ha incrementado de forma notable en las últimas décadas, convirtiéndose en una arena compleja y diversificada, y en su interior pueden encontrarse posiciones muy diversas, algunas se expresan desde la autonomía periodística (esta figura se acercaría, por tanto, al modelo del intelectual clásico) y otras dependen de los intereses de los poderosos.

Además de los medios de comunicación de masas, otros espacios orientados 
a la intervención pública, y con una influencia creciente, son los think tanks, que han sido estudiados con precisión, desde la nueva sociología de los intelectuales, por Thomas Medvetz (2012). Medvetz parte de un análisis sociológico muy similar al desarrollado por Jacobs y Townsley, Su objetivo es estudiar la estructura interna y la dinámica del «espacio de los think tanks», entendido como un campo de conocimiento experto, es decir, un "conjunto de dilemas y restricciones al que se enfrentan los grupos de expertos en sus encuentros con los consumidores o entre ellos mismos» (Medvetz, 2012: 85). El sociólogo norteamericano muestra la estructura del espacio de los think tanks en Estados Unidos según dos ejes fundamentales: un eje horizontal que se refiere a la tensión entre "autonomía» y "heteronomía», y un eje vertical que se basa en la elección entre "apertura" y "clausura». Al igual que Jacobs y Townsley, Medvetz asegura que, a diferencia de los teóricos clásicos defensores de la autonomía y la clausura del campo intelectual, se trata de una cuestión compleja y, al final, los autores se sitúan en diferentes coordenadas dentro de ambos ejes. Frente a las tesis sobre la desaparición de los intelectuales, Medvetz asegura que, actualmente, la acción intelectual se concentra sobre todo en este ámbito.

\section{Conclusión}

La sociología de los intelectuales ha sufrido un intenso proceso de redefinición en las últimas décadas. En gran medida, Bourdieu y Collins son los máximos responsables de las nuevas tendencias de investigación, aportando conceptos teóricos y metodologías que hoy en día son muy influyentes. Sin embargo, sus propuestas específicas del intelectual se mantienen dentro de cánones bastante clásicos: tanto los "campos culturales especializados" como las "comunidades intelectuales" remiten directamente a las concepciones tradicionales de la alta cultura, que establecen un rígido cordón sanitario entre prácticas culturales legítimas e ilegítimas. Desde nuestro punto de vista, la sociología de los intelectuales consagrados y la sociología de las comunidades intelectuales han seguido el camino delineado por Collins y Bourdieu. Tomando en consideración las profundas transformaciones que se están produciendo en el campo cultural (que incluyen, por ejemplo, el proceso de digitalización) creemos que es necesario superar (o al menos expandir) las concepciones clásicas del intelectual y definir los nuevos contornos que está adquiriendo dicho actor social. En este sentido, la reciente "sociología de las intervenciones públicas" está abriendo nuevos espacios de investigación que podrían ser muy fructíferos. Desde este ámbito de investigación podría reconstruirse la agenda de investigación e incluir una serie de cuestiones clave que no suelen tomarse en consideración: a) la aparición de nuevos contextos de acción intelectual; b) la diversificación de los actores que realizan acciones intelectuales; c) las nuevas estrategias (que implicarán usos novedosos de los medios) de la acción intelectual; y d) las nuevas mentalidades y configuraciones simbólicas que incitan a la acción intelectual. Una sociología de los intelectuales así reformulada podría ayudarnos a comprender la construcción de la opinión asociada a los procesos de cambio social. 


\section{Referencias bibliográficas}

Aron, Raymond (1955). El opio de los intelectuales. Barcelona: RBA, 2011.

BAERT, Patrick e IsAAC, Joel (2011). «Intellectuals and society: Sociological and historical perspectives». En: Delanty, Gerard y Turner, Stephen (eds.). Handbook of Contemporary Social and Political Theory. Londres: Routledge.

BaUman, Zygmunt (1987). Legislators and interpreters: On modernity, postmodernity and intellectuals. Cambridge: Polity Press.

Bell, Daniel (1960). The end of ideology: On the exhaustion of political ideas in the fifties. Nueva York: The Free Press, 2000.

- (1973). El advenimiento de la sociedad postindustrial. Madrid: Alianza, 2006.

Benda, Julien (1927). La traición de los intelectuales. Madrid: Galaxia Gutenberg, 2008.

Boschetti, Laura (1985). Sartre et «Les Temps Modernes». París: Minuit.

Boudon, Raymond (1981). "L'intellectuel et ses publics: Les singularités françaises». En: Reymaud, Jean-Daniel y Grafmeyer, Yves (comp.). Français, qui êts-vous? París: La Documentation Française.

Bourdieu, Pierre (1971). "Le marché des biens symboliques». L'Année Sociologique, 22, 49-126.

- (1983). «The field of cultural production, or the economic world reversed». Poetics, $12,211-356$.

- (1984). Homo academicus. Madrid: Siglo XXI, 2008.

- (1988), La ontología política de Martin Heidegger. Barcelona: Paidós.

- (1995). Las reglas del arte: Génesis y estructura del campo literario. Barcelona: Anagrama.

- (1997). Sobre la televisión. Barcelona: Anagrama.

CAmic, Charles (1987). «The making of a method: A historical reinterpretation of the early Parsons». American Sociological Review [en línea], 52 (4), 421-439. <http://dx.doi.org/10.2307/2095289>.

- (1992). "Reputation and predecessor selection: Parsons and the institutionalists». American Sociological Review [en línea], 57 (4), 421-445. <http://dx.doi. org/10.2307/2096093>.

Camic, Charles y Gross, Neil (2001). "The new sociology of ideas». En: Blau, Judith (ed.). The Blackwell companion to sociology. Oxford: Blackwell.

Casanova, Pascale (2003). La República Mundial de las Letras. Barcelona: Anagrama.

Charle, Christophe (1990). Naissance des intellectuels, 1880-1900. París: Éditions de Minuit.

- (1996). Les intellectuels en Europe au siècle XIX: Essai d'histoire comparée. París: Seuil.

Сномкку, Noam (1969). American power and the new mandarins: Political and historical essays. Nueva York: Pantheon Books.

Collins, Randall (1975). Conflict sociology: Towards an explanatory science. Nueva York: Academic Press.

- (1998). The sociology of philosophies: A global theory of intelectual change. Boston: The Belknap Press.

Coser, Lewis (1965). Hombres de ideas: El punto de vista de un sociólogo. México: Fondo Económico de Cultura.

Cusset, François (2008). French Theory: Foucault, Derrida, Deleuze \& Cía y las mutaciones de la vida intelectual en Estados Unidos. Barcelona: Melusina.

DeBray, Régis (1979). Teachers, writers and celebrities: The intellectuals in modern France. Londres: Verso. 
DJilas, Milovan (1957). The new class: An analysis of the communist system. Nueva York: Praeger.

Dosse, François (2003). La marcha de las ideas: Historia de los intelectuales. Historia intelectual. Valencia: Publicacions de la Universitat de València.

Elias, Norbert (2002). Mozart: Sociología de un genio. Barcelona: Península.

Epstein, Steven (1996). Impure science: AIDS, activism and the politics of knowledge. Berkeley: University of California Press.

Eyal, Gil y Buchiolz, Larissa (2010). «From the sociology of intellectuals to the sociology of interventions». Annual Review of Sociology [en línea], 36, 117-137. <http://dx.doi.org/10.1146/annurev.soc.012809.102625>.

Ferry, Luc y Renaut, Alain (1985), La pensé 68. Paris: Gallimard.

Foucault, Michel; Gordon, Colin et al. (1980). Power/knowledge: Selected interviews and other writings 1972-1977. Harlow (Essex): The Harvester Press.

Frickel, Scott y Gross, Neil (2005). "A general theory of scientific/intellectual movements». American Sociological Review [en línea], 70 (2), 204-232. <http://dx.doi.org/10.1177/000312240507000202>.

Gella, Aleksander (ed.) (1976). The intelligentsia and the intellectuals: theory, method and case study. Beverly Hills: Sage.

Gouldner, Alvin (1970). The coming crisis of western sociology. Nueva York: Basic Books.

- (1979). The future of intellectuals and the rise of the new class. Nueva York: Seabury.

- (1985). Against fragmentation: The origins of marxism and the sociology of intellectuals. Nueva York: Oxford University Press.

Gramsci, Antonio (1926-1937). Antonio Gramsci: Antología. Madrid: Siglo XXI, 2010.

Gross, Neil (2008). Richard Rorty: La forja de un filósofo americano. Valencia: Publicacions de la Universitat de València.

Jacobs, Ronald y Townsley, Eleanor (2011). The space of opinion: Media intellectuals and the public sphere. Oxford: Oxford University Press.

JACOBY, Russell (1987). The last intellectuals: American culture in the age of academe. Nueva York: Basic Books.

JoAs, Hans y KNöBL, Wolfgang (2009). Social theory: Twenty introductory lectures. Oxford: Oxford University Press.

Judt, Tony (2007). Pasado imperfecto: Los intelectuales franceses, 1944-1956. Madrid: Taurus.

Kadushin. Charles (1974). The American intelectual elite. Londres: Little, Brown and Company.

Kauppi, Niilo (1996). French intellectual nobility: Institutional and symbolic transformations in the post-Sartrian era. Albany: SUNY Press.

Keck, Margaret y Sikкink, Kathryn (1998). Activists beyong borders: Advocacy networkws in international politics. Ithaca, Nueva York: Cornell University Press.

KonRad, György y SzelenYI, Iván (1979). The intellectuals on the road to class power. Nueva York: Harcourt.

Kurzman, Charles y Owens, Lynn (2002). "The sociology of intellectuals». Annual Review of Sociology [en línea], 28, 63-90. <http://doi.org/10.1146/annurev.soc.28.110601.140745>.

Lamont, Michelle (1987). "How to become a dominant French philosopher: The case of Jacques Derrida». The American Journal of Sociology [en línea], 93 (3), 584-622. <http://dx.doi.org/10.1086/228790>. 
- (2010). How professors think: Inside the curious world of academic judgement. Cambridge (MA): Harvard University Press.

LEPENIES, Wolf (1994). Las tres culturas: La sociología entre la literatura y la ciencia. Madrid: Fondo de Cultura Económica.

Lipset, Seymour (1960). Political man: The social bases of politics. Garden City: Doubleday \& Company.

Lyotard, Jean-François (1984). Le tombeau des intellectuales et autres papiers. París: Gallimard.

MannheIm, Karl (1934). Ideología y Utopía: Introducción a la sociología del conocimiento. México. Fondo de Cultura Económica, 2004.

Medvetz, Thomas (2012). Think tanks in America. Oxford: Oxford University Press.

Merton, Robert (1968). Teoría y estructuras sociales. México: Fondo Económico de Cultura.

Misztal, Barbara y Freundlieb, Dieter (2003). «The curious historical determinism of Randall Collins». European Journal of Sociology [en línea], 42 (2), 247-269. <http://dx.doi.org/10.1017/s0003975603001267>.

Moreno Pestaña, José Luis (2008). Filosofía y sociología de Jesús Ibáñez. Madrid: Siglo XXI.

- (2013). La norma de la filosofía. Madrid: Biblioteca Nueva.

Parsons, Talcott (1968). La estructura de la acción social: Estudio de teoría social, con referencia a un grupo de recientes escritores europeos. 2 vols. Madrid: Guadarrama.

— «The intellectual: A social category role». En: On intellectuals: Theoretical studies and case studies, Nueva York: Doubleday.

Pecourt, Juan (2008). Los intelectuales y la transición política: Un estudio del campo de las revistas politicas en España. Madrid: CIS.

Picó, Josep y Pecourt, Juan (2008). «El estudio de los intelectuales: Una reflexión». Revista Española de Investigaciones Sociológicas, 123, 35-58.

Posner, Richard (2001). Public intellectuals: A study of decline. Cambridge (MA): Harvard University Press.

Renaut, Alain y Ferry, Luc (1985). Le pensée de 68: Essai sur l'anti-humanisme contemporain. París: Gallimard.

RiefFeL, Rémy (1993). La tribu des clercs: Les intellectuels sous la V Reppublique. París: CNRS Éditions.

Ringer, Fritz (1969). The decline of the German mandarins: The German academic community, 1890-1933. Cambridge (MA): Harvard University Press.

Rorty, Richard (1989). La filosofía y el espejo de la naturaleza. Madrid: Cátedra.

Rubinich, Lucas (2001). La conformación de un clima cultural: Neoliberalismo y universidad. Buenos Aires: EUDEBA.

SAID, Edward. (1994). Representations of the intellectual: the 1993 Reith Lectures. Nueva York: Pantheon Books.

SAPIRO, Giselle (2009a). «Modèles d'intervention politique des intellectuels: Le cas français». En: Actes de la Recherche en Ciences Socials [en línea], 176-7, 8-31. <http://dx.doi.org/10.3917/arss.176.0008>.

- (2009b). L'espace intellectuel en Europe: De la formation des États-nations à la mondialisation XIX-XXI siècle. París: La Découverte.

SArtre, Jean-Paul (1972). Plaidoyer pour les intellectuels. París: Gallimard.

SHILs, Edward (1972). Intellectuals and the powers and other essays. Chicago: Chicago University Press. 
SkINNER, Quentin (2002). Visions of politics. Vol. 1. Cambridge: Cambridge University Press.

- (2008). Hobbes and Republican liberty. Cambridge: Cambridge University Press.

SwarTZ, David (1997). Culture and power: The sociology of Pierre Bourdieu. Chicago: Chicago University Press.

VÁzquez García, Francisco (2009). La filosofía Española: Herederos y pretendientes. Madrid: Abada.

Wright Mills, Charles (2008). The politics of truth: Selected writings of C. Wright Mills. Oxford: Oxford University Press. 